\title{
Interação Mãe-Criança e Desenvolvimento da Linguagem: A Influência da Depressão Pós-Parto
}

\author{
Beatriz Servilha \\ Vera Silvia Raad Bussab \\ Universidade de São Paulo \\ São Paulo, SP, Brasil
}

\begin{abstract}
RESUMO
O objetivo desta pesquisa foi verificar a influência da depressão pós-parto e do contexto afetivo de criação no desenvolvimento da linguagem de crianças de 36 meses. Foram analisadas 80 díades mães-crianças atendidas pelo sistema público de saúde: dentre as mães, 30 apresentaram indicativos de DPP. Avaliou-se o aspecto pragmático do desenvolvimento de linguagem, em filmagens de quinze minutos de brincadeira livre com a mãe, em função de informações colhidas desde o nascimento. O resultado do teste foi analisado quanto aos atos e meios comunicativos realizados durante a interação. A partir da análise dos dados, observou-se que, embora a maior parte das crianças apresentou um desempenho abaixo do esperado para a idade, as crianças do grupo controle verbalizaram mais para se comunicar que aquelas do grupo com DPP, que usaram mais gestos durante a interação. Concluiu-se que os aspectos relacionados ao s fatores da DPP influenciaram, de forma direta na interação diádica e no desenvolvimento da linguagem infantil.
\end{abstract}

Palavras-chave: Depressão pós-parto; Desenvolvimento da linguagem; Relações mãe-filho.

\section{ABSTRACT}

\section{Mother-Child Interaction and Language Development: The Influence os Postpartum Depression}

The aim of this study was to analyze the influence of the affective upbringing context and postpartum depression (PPD) on the language development of thirty-six-month-old child. We evaluated the pragmatic development aspect of child language in 80 mother-child dyads, filmed during a free play session of fifteen minutes, in function of the information collected since birth. The test result was analyzed, considering the communicative acts and means accomplished during social interaction. Based on the data analysis, it was observed that, although most of the children present lower performance than expected for this age, children from the control group used more verbal interaction than the PPD group, while children from the PPD group used more gestural communication. In conclusion, the aspects related to the PPD factors directly influenced the dyads' interaction and the children's language development.

Keywords: Postpartum depression; Language development; Mother-child relationship

\section{RESUMEN}

Interacción Madre-Hijo y en el Desarrollo del Lenguaje: La Influencia de la Depresión Posparto

El objetivo de esta investigación fue averiguar la influencia de la depresión pós-parto (DPP) y el contexto afectivo de la creación en el desarrollo del lenguaje en niños de 36 meses. Hemos analizado 80 diades de madres-niños contestadas por el sistema público de salud,desde la gestación: entre las madres, son 30 las que presentaban indicativos de DPP. Fue evaluado el aspecto pragmático del desarrollo del lenguaje, en imágenes de los 15 minutos de juego libre con la madre, a la luz de la información recogida desde el nacimiento. El resultado de la prueba fue analizada por la comisión de actos comunicativos y medio realizado durante la interacción. Del análisis de los datos, se observó que, a pesar de que la mayoría de los niños presenta un rendimiento por debajo de lo esperado para la edad, los niños en el grupo control verbalizan más para comunicarse que en el grupo con DPP, que utilizan más los gestos durante la interacción. Se llegó a la conclusión de que los aspectos relacionados con los factores de DPP influyen directamente en la interacción diádica y en desarrollo del lenguaje infantil.

Palabras clave: Depresión posparto; Desarrollo del lenguaje; Relaciones madre-hijo. 


\section{INTRODUÇÃO}

Há reconhecimento convergente de vários setores do estudo da ontogênese humana de que a interação é condição necessária não só para a inserção social do indivíduo, mas também para seu desenvolvimento cognitivo pleno. Faz parte de um processo evolutivo, histórico e cultural (Seidl-de-Mora e Ribas, 2004; Vieira e Prado, 2004; Yamamoto e Lopes, 2004; Seidlde-Moura, 2009; Pessôa, 2009; Murray et al, 2010). A interação é entendida como condição de possibilidade da existência do sujeito, pois este só se constitui como ser autônomo na relação com os outros. Igualmente, é constitutiva da ontogênese dos processos cognitivos. O diálogo é a expressão viva do relacionamento interpessoal, delimitando os enunciados e organizando o processo de enunciação. A palavra é sempre dialógica, pois evoca significações que a antecedem, ao mesmo tempo que desencadeia reações subsequentes. Dessa forma, a atividade discursiva no processo de interação é necessário para a subjetivação e importante na vida social humana (Colaço, 2004).

A perspectiva sócio pragmática insere as interações sociais como uma das bases fundamentais do desenvolvimento inicial da comunicação e da linguagem. Dentro da abordagem interacionista e evolucionista do desenvolvimento, considera-se um conjunto de predisposições genéticas ou capacidades biológicas de nossa espécie tanto para reconhecimento de co-específicos e estabelecimento de trocas sociais, quanto para tratamento de estímulos específicos como a voz humana, presentes no nascimento. Nesse desenvolvimento são indispensáveis os fatores externos ambientais, para que a fala apareça. A separação entre biologia/natureza e cultura não faz sentido, pois é a troca entre estes segmentos que a exposição aos estímulos linguísticos ocorre. Nesse processo destaca-se o papel da função materna (Vieira e Prado, 2004; Pessôa, 2009).

A habilidade materna em perceber, interpretar e responder às necessidades comunicativas da criança assume posição central na construção de uma relação de mutualidade. $\mathrm{O}$ vínculo afetivo com o bebê pode ser desenvolvido ainda na gravidez, mas tende a aumentar consideravelmente após o nascimento. As primeiras fases após o parto são importantes no processo de desenvolvimento e fortalecimento dos vínculos afetivos entre a mãe e o filho (Thomaz et al., 2005; Araújo, 2007). A responsividade materna, ou seja, a prontidão e a resposta apropriada da mãe às atividades comunicativas e exploratórias das crianças auxilia o processo de aquisição da fala e do vocabulário da criança. Justo por isto que a mãe é coautora no desenvolvimento comunicativo-linguístico de seu filho. A fala materna promove o desenvolvimento da fala infantil, a partir da conexão semântica entre os interlocutores e os diferentes empregos sintáticos apresentados às crianças, estimulando-as a aprenderem significados verbais. $\mathrm{O}$ entrosamento linguístico favorece o desempenho desejado em níveis de emissão e apreensão semântica. A responsividade materna, por sua vez, é modulada por fatores múltiplos e variados, tanto das características da mãe e do ambiente sócioafetivo, bem como pelas características dos bebês, que têm forte influência no comportamento da mãe (Vieira e Prado, 2004).

Taylor et al. (2008) verificaram o quanto a sensibilidade e a responsividade materna contribuem para o desenvolvimento da linguagem durante o processo de interação entre da mãe com a criança. A pesquisa foi um estudo longitudinal com 62 díades, verificando a interação aos $6,12,18$ e 24 meses e o desenvolvimento da linguagem da criança. Observou-se, neste trabalho, que o desenvolvimento da linguagem das crianças mostrou-se muito sensível à variedade das condições ambientais, incluindo a disponibilidade e riqueza da estimulação linguística e cognitiva transmitida por pais sensíveis e responsivos.

Esta responsividade materna pode ser prejudicada quando a mãe apresenta depressão pós-parto (DPP). A depressão pós-parto é um problema de saúde pública que afeta tanto a saúde quanto a interação diádica. Caracteriza-se por um episódio depressivo ou uma soma de episódios após o nascimento de um filho, que se iniciam geralmente entre a quarta e oitava semanas após o parto, alcançando sua intensidade máxima nos seis primeiros meses (Murray et al., 2010, Schwengber e Piccinini 2003). Estima-se que 10 a 15\% das mulheres apresentem sintomas depressivos durante esse período após o parto. Porém vários estudos mostraram variações neste índice, por questões metodológicas e contextuais (Silva,2008). Trabalhos utilizando a Escala de Depressão Pós-Parto de Edimburgo relatam prevalências de 12\% (Da Silva et al., 1998), 22,2\% (Zaconeta, Motta,França, 2004), 28\% (Fonseca, Silva, Otta, 2010), 36,8\% (Skafuzca, 2000) e 37,1\% (Cruz, Simões, Faisal-Cury, 2005) de depressão pós-parto na população brasileira.

Darcy et al. (2011) mostraram que os sintomas característicos da DPP são: alteração do humor para depressivo/irritável ou desinteresse/falta de motivação; redução de energia e anedonia (redução ou ausência da capacidade de sentir prazer na vida ou no lazer), choro frequente, sentimentos de culpa, alterações no sono, temos em machucar o filho, diminuição de apetite e libido, diminuição do nível e funcionamento mental, idéias suicidas, obsessivas ou supervalorizadas. 
Fatores apontados como de risco para o desenvolvimento de DDP são: a) depressão e ansiedade pré-natal; b) autoestima baixa; c) dificuldade em lidar com o bebê; d) alto nível de estresse; e) pouco suporte social; f) relação marital pobre; g) história de depressão; e h) presença de melancolia. Outros fatores também se mostraram associados com a depressão pós-parto: ser mãe solteira, pertencer a um baixo nível sócio-econômico, ter uma gestação não planejada ou não desejada, complicações devidas à gestação ou parto, idade e nascimentos de pré-termos e de gêmeos (Murray et al., 2010; Darcy et al., 2011).

O suporte social, de acordo com Konradt et al. (2011) pode ser entendido como o apoio emocional ou prático fornecido pela família e/ou amigos, na forma de afeto, companhia, assistência e informação, incluindo tudo o que gera no indivíduo a sensação de ser amado, estimado, cuidado, valorizado e seguro. Além disso, é um conjunto de fatores socioambientais que atuam de forma protetora, habilitando os indivíduos a lidarem mais adequadamente com os estressores ambientais. Os autores ainda relatam que, quando essa habilidade falha ou não é suficiente, a adaptação da mulher ao seu novo papel social torna-se mais difícil, por isso sua direta ligação com os sintomas depressivos e inúmeros estudos a respeito da ligação entre suporte social e depressão pós-parto.

Schwinger e Piccinini (2005) também relatam a influência do apoio social, principalmente do companheiro, nos sintomas depressivos maternos. Os autores investigaram a experiência da maternidade no contexto da depressão materna no final do primeiro ano de vida do bebê. Foi constatado, no estudo, que somente as mães que apresentavam sintomas depressivos apresentaram impressões ambivalentes a respeito do companheiro como pai e, apesar de reconhecerem a presença de uma rede de apoio nos cuidados com o bebê, este grupo demonstrou maior insatisfação com o apoio recebido pelo companheiro e por outras pessoas. Impressões positivas em relação à ajuda com a criança foram relatadas apenas por mães sem indicadores de depressão.

Silva (2008), em sua dissertação de mestrado, realizou um estudo longitudinal sobre a prevalência e fatores de risco para a depressão pós-parto em mães de baixa renda. Observou-se que as mães com maiores escores de DPP foram aquelas que apresentavam percepção de baixo apoio social na gestação e maiores dificuldades de contar com a rede de apoio no cuidado com a criança. Na fase do pós-parto, de acordo com Silva et al. (2010), o tipo e a natureza do suporte recebido são fatores possíveis de contribuir para melhor adaptação e alcance do papel materno.
A DPP pode ser prejudicial ao vínculo mãe-bebê e é considerada um fator de risco para o desenvolvimento infantil, pois compromete a disponibilidade cognitiva e emocional da mãe pela falta de habilidade em lidar com os próprios problemas e a falta de persistência necessária para estabelecer uma interação sensível com a criança (Frizzo e Piccinini, 2005; Schmidt et al., 2005; Murray, 2010). Darcy et al. (2011) constataram que índices de depressão elevados durante o período pós-parto podem trazer implicações para o trabalho físico e mental da mãe e, consequentemente, para sua qualidade de vida.

A interação mãe-bebê, no caso de DPP, pode apresentar mais caracteristicamente: expressões negativas mediante características emocionais e comportamentais dos bebês; menor afetividade e maior ansiedade nos cuidados maternos; níveis maiores de hostilidade, mais rejeição, negligência e agressividade.

Os bebês, por sua vez, tendem a exibir mais afeto negativo (expressões de tristeza, raiva ou tendência à irritabilidade); ser menos responsivos nas relações interpessoais (menos vocalizações); ter o desenvolvimento motor mais precário; apresentar, em idade pré-escolar, dificuldades para se envolver e manter uma interação social, tendo déficits na regulação dos seus estados afetivos. ter maiores dificuldades alimentares e de sono (Motta, 2005; Schmidt et al., 2005, Frizzo e Piccinini, 2005, Lopes et al., 2010).

As mães deprimidas passam menos tempo olhando, tocando, falando com seus bebês, mostram menos responsividade, espontaneidade e menores índices de atividade. A depressão pós-parto contribui para que os comportamentos afetivos e de atenção da díade tornemse assincrônicos, na medida em que a mãe encontrase não-responsiva afetivamente. Dessa forma, a DPP afeta a criança pela alteração dos modelos de interação diádica. A exposição da criança a estilos de interação não adequados pode representar diferentes tipos de risco para o desenvolvimento afetivo e cognitivo da criança, como: depressão, baixa estima, dificuldades escolares e de interação social, alterações linguísticas, entre outras (Seidl-de-Moura, Ribas, 2004, Schwengber, Piccinini, 2003).

Com ênfase no entendimento dos fatores relevantes para o desenvolvimento infantil o presente projeto está inserido no Projeto Temático FAPESP (nº 06/59192), conhecido como Projeto Ipê, “Depressão pós-parto como um fator de risco para o desenvolvimento do bebê: estudo interdisciplinar dos fatores envolvidos na gênese do quadro e em suas consequências". Trata-se de acompanhamento longitudinal com o compromisso de favorecer estudos na área, aprofundando a compreensão da origem da depressão pós-parto, além de analisar 
a incidência do quadro relacionado às condições de suporte social (material, afetivo e emocional), à fase da vida reprodutiva da mãe, às questões hormonais, de desenvolvimento e à vinculação diádica. Igualmente, avalia as implicações potenciais para a mãe e para o desenvolvimento global do bebê, através de medidas dos índices de desenvolvimento geral e neuro-psicomotor, e dos níveis de cortisol. Além disso, o projeto tem como um de seus focos o momento da mulher frente à maternidade e seus desafios. Envolve, portanto, áreas de pesquisa básica e clínica, com diversidade de profissionais de várias especialidades que se relacionam com o tema DPP, destacando a relevância dos subsídios que podem fornecer para práticas de intervenção precoce.

Este estudo objetivou verificar as influências da DPP e do contexto afetivo de criação no desenvolvimento da linguagem de crianças de 3 anos. A análise destes efeitos foi feita dentro de um contexto mais amplo de variáveis relacionadas à composição familiar e ao apoio social, o que permitiu aprofundar a compreensão dos processos subjacentes.

\section{OBJETIVO}

Verificar a influência da depressão pós-parto e do contexto afetivo de criação no desenvolvimento da linguagem de crianças de 36 meses de idade.

\section{MÉTODO}

\section{Sujeitos}

Foram analisadas oitenta mães e crianças de três anos, participantes do projeto já referido. Das crianças participantes, 31 eram do sexo masculino e 49 do sexo feminino e apresentavam idade entre de 3 anos e 3 anos e 5 meses. Com relação às mães, 30 apresentaram indicativos de DPP, avaliada pela Escala de Depressão Pós-parto de Edinburgh (Cox, Holden, \& Sagovsky, 1987; validada por Santos, Martins, \& Pasquali, 1999).

\section{Instrumentos}

Foram utilizados na pesquisa os seguintes instrumentos:

- Escala de Depressão Pós-natal de Edinburgh (EDPE), de Cox, Holden e Sagovsky (1987), validada no Brasil por Santos et al. (1999). Utilizada para rastrear a prevalência de depressão pós-parto (escores $\geq 12$ ). A EDPE é um instrumento de autopreenchimento composto por 10 enunciados (ex: Eu tenho sido capaz de rir e achar graça das coisas; Eu tenho me culpado sem necessidade quando as coisas saem erradas; Eu tenho me sentido esmagada pelas tarefas e acontecimentos do meu dia-a-dia; Eu tenho me sentido triste ou arrasada) cujas opções são pontuadas ( 0 a 3 ) de acordo com a presença ou intensidade do sintoma. A EDPE foi aplicada entre a 9 ${ }^{\underline{a}}$ e a $12^{\underline{a}}$ semanas após o parto, no IPUSP;

- Entrevistas semiestruturadas. Foram realizadas entrevistas semiestruturadas durante o último trimestre de gestação (nas UBS), no período de internação após o parto (no Hospital Universitário) e aos 4 meses no Laboratório de Observação do Comportamento do IPUSP. As entrevistas tiveram por objetivo levantar dados sobre o final da gestação, o parto e eventuais dificuldades no cuidado com o bebê. Na entrevista foram obtidos dados pessoais da participante (idade, escolaridade, renda familiar, utilização de métodos anticoncepcionais, etc), sobre o pai do bebê (idade, escolaridade, renda, etc.) e sobre aspectos emocionais da gravidez (irritabilidade, choro, alegria, tristeza, etc.). Também foram levantadas informações a respeito do apoio recebido pela mãe por parte de familiares, amigos e, principalmente, do companheiro nos cuidados com o bebê. Eventuais episódios de depressão e/ou DPP sofridos pela participante ou parentes de 1ㅇ Grau maternos e paternos foram registrados.

As mães participantes do projeto foram divididas de acordo com a apresentação ou não de sintomas de depressão pós-parto, a partir da pontuação obtida na Escala de Depressão Pós-Parto de Edimburgo - EDPE. (Cox, Holden, \& Sagovsky, 1987).

Com a criança foi realizada uma avaliação da pragmática da linguagem, através do teste fonoaudiológico $A B F W$ (Andrade, Befi-Lopes, \& Fernandes Wertzner, 2000). O teste possui protocolo específico para avaliar atos comunicativos totais realizados pela mãe e pela criança; atos comunicativos somente da criança e atos comunicativos realizados por minuto (a quantidade de atos por minuto mostra, dentro dos parâmetros apontados pela autora, se a criança está com o seu desempenho dentro do esperado para a idade). Para amostras correspondentes da faixa etária estudada, os autores indicam como referência, aproximadamente, seis a oito atos por minuto.

Também são avaliados os meios comunicativos utilizados: verbais (VE), aqueles que envolvem pelo menos $75 \%$ de fonemas da língua; e vocais (VO), referentes a todas as outras emissões e gestuais $(G)$, os quais envolvem movimentos de corpo e rosto (Fernandes, F.D.M.(2000). 


\section{Procedimento}

A pesquisa foi aprovada pelo comitê de ética do HU e pelo Comitê de Ética em Pesquisa com Seres Humanos do IPUSP, seguindo todos os princípios éticos. Os participantes que fazem parte deste estudo, aceitaram participar da pesquisa e assinaram o Termo de Consentimento Livre e Esclarecido.

Para avaliação do aspecto pragmático da linguagem da criança, a mãe e criança foram filmadas interagindo em brincadeira livre por 15 minutos com os brinquedos: casinha de madeira, bonecos de pano, móveis e utensílios da casa (cama, mesa, cadeiras, etc.). Essa atividade foi realizada em uma sala específica, preparada para o procedimento. A mãe e a criança ficam a sós na sala, filmadas por duas câmeras. Todas as comunicações filmadas foram transcritas nos protocolos do teste de ABFW, juntamente com os comportamentos e fatos observados por uma profissional com formação em psicologia, responsável pelas filmagens do Projeto Ipê. A transcrição foi realizada de forma manual pela autora do trabalho e duas alunas de psicologia, bolsista de iniciação científica dentro do projeto longitudinal. A análise das filmagens foi realizada pela autora do trabalho, fonoaudióloga, especialista em linguagem infantil e treinada para utilizar o instrumento acima descrito.

\section{Análise dos dados}

Foram analisadas as quantidades de atos comunicativos totais (a soma de atos realizados pela mãe e pela criança), a quantidade de atos comunicativos realizados somente pela criança. Com este, calculou-se a ocorrência de atos comunicativos por minuto (para verificação do desempenho da criança no teste). Verificou-se também as ocorrências dos meios (vocal, verbal e gestual) durante o discurso da criança. Estes aspectos foram analisados em conjunto com aspectos socioafetivos do desenvolvimento, tais como a presença ou ausência de DPP, trabalho materno, apoio social e frequência da criança à creche. Para tal foram utilizados percentuais descritivos e testes estatísticos, como: testes qui-quadrado de contingência, testes de média a fim de verificar a diferença entre mais de dois (ANOVA) grupos em função de uma ou mais variáveis contínuas dependentes e técnicas multivariadas (os dados acima descritos são variáveis consideradas correlacionadas) como análise de componentes principais, cluster e análise de correspondência.

Identificado o número de grupos através do Método Hierárquico Ward, analisou-se os dados utilizando o Método de Agrupamento Não Hierárquico K-médias a fim de melhor classificar as observações dentro de cada cluster. Assim, os clusters foram caracterizados da seguinte forma:

- cluster 1: crianças com os melhores desempenhos em todos os meios considerados;

- cluster 2: crianças com bom desempenho, com uma exceção no gestual; e

- cluster 3: crianças com desempenho ruim.

O nível de significância adotado foi de 5\%.

\section{RESULTADOS}

\section{Desempenho geral das crianças no teste ABFW}

Quanto aos atos comunicativos totais, obteve-se média de 179 atos comunicativos totais, sendo 89,2 a média realizada somente pelas crianças. Observouse correlação significativa entre estas duas variáveis $(F=0,93 ; p<0,0001)$, ou seja, quanto mais a criança falar, maior será a interação diádica.

Metade das crianças avaliadas (52,5\%) ficou abaixo do esperado para a idade, uma vez que o instrumento sugere entre 6 a 8 atos comunicativos por minuto). A Tabela 1 mostra os desempenhos esperados para a idade e os desempenhos apresentados pelos grupos. Quando comparado o desempenho com relação aos clusters, verificou-se que a maioria das crianças dos clusters 1 e 2 obtiveram desempenho esperado para a idade, ( $81,25 \%$ e $82,75 \%$ respectivamente). O cluster 3 foi o grupo com pior desempenho, em que todas as crianças estiveram abaixo do esperado para a idade. $\mathrm{O}$ meio verbal foi usado por todas as crianças (média de 77,2 verbalizações), e o gestual por $90 \%$ destas (média de 10,6 gestos). O menos utilizado foi o vocal, por $82,5 \%$ das crianças com uma (média de 4,3 vocalizações). Foram observadas correlações positivas entre os atos comunicativos totais e da criança com o meio verbal ( $F=0,88$ e 0,$92 ; p<0,0001$, respectivamente), ou seja, quanto maior a comunicação, mais o meio verbal foi utilizado.

\section{Pragmática e contexto de desenvolvimento}

O desempenho das crianças no teste foi verificado em relação aos dados do ambiente de criação (frequência na creche, apoio social, trabalho materno), através do teste MANOVA. Houve apenas efeito da creche e da ajuda no meio gestual $(F=0,046 ; p<0,05)$, ou seja, as crianças que não frequentam a creche e as mães possuem apoio social utilizam mais gestos em sua comunicação, conforme a Tabela 1 .

As vocalizações, mais uma vez, foram utilizadas com menor frequência por ambos os grupos. 
TABELA 1

Média e desvio padrão de desempenho no teste com relação a variáveis socioafetivas de criação

\begin{tabular}{lcccccc}
\hline \multirow{2}{*}{ Variáveis } & \multicolumn{2}{c}{ Creche } & \multicolumn{2}{c}{ Trabalha } & \multicolumn{2}{c}{ Apoio } \\
\cline { 2 - 6 } & \multicolumn{1}{c}{ Sim } & Não & Sim & Não & Sim & Não \\
\hline ACT & $90,6(60,5)$ & $85,7(45,2)$ & $174,8(57,2)$ & $182,3(56,1)$ & $88,4(54,8)$ & $90,6(62,4)$ \\
ACC & $180,9(31)$ & $174,5(22,1)$ & $86,4(30,9)$ & $91,2(27,3)$ & $178,9(28,6)$ & $179,3(30,6)$ \\
Verbal & $78,5(28,9)$ & $74(20,7)$ & $74,3(26,8)$ & $79,4(26,8)$ & $77(26,4)$ & $78(29,4)$ \\
Vocal & $4,3(4)$ & $4,2(4,6)$ & $4,8(4,7)$ & $4,8(3,9)$ & $3,6(3,6)$ & $4,8(4,3)$ \\
Gestual & $10(9,3)$ & $11,3(10,8)$ & $11,3(10,6)$ & $9,8(9,1)$ & $12,3(9,8)$ & $12,3(9,9)$ \\
\hline
\end{tabular}

ACT: atos comunicativos totais; ACC: atos comunicativos somente das crianças; Verbal: quantidade de verbalizações realizadas somente pelas crianças; Vocal: quantidade de vocalizações realizadas somente pelas crianças; Gestual: quantidade de gestos realizados somente pelas crianças.

TABELA 2

Análise comparativa dos dados da anamnese entre clusters

\begin{tabular}{|c|c|c|c|c|c|c|c|c|c|c|c|c|}
\hline \multirow{3}{*}{ Clusters } & \multicolumn{4}{|c|}{ Creche } & \multicolumn{4}{|c|}{ Trabalha } & \multicolumn{4}{|c|}{ Apoio } \\
\hline & \multicolumn{2}{|c|}{ Sim } & \multicolumn{2}{|c|}{ Não } & \multicolumn{2}{|c|}{ Sim } & \multicolumn{2}{|c|}{ Não } & \multicolumn{2}{|c|}{ Sim } & \multicolumn{2}{|c|}{ Não } \\
\hline & $N$ & $\%$ & $N$ & $\%$ & $N$ & $\%$ & $N$ & $\%$ & $N$ & $\%$ & $N$ & $\%$ \\
\hline Cluster 1 & 11 & 68,75 & 5 & 31,25 & 6 & 37,5 & 10 & 62,5 & 7 & 43,7 & 7 & 56,2 \\
\hline Cluster 2 & 23 & 79,3 & 6 & 20,7 & 12 & 41,3 & 17 & 58,6 & 21 & 72,4 & 8 & 27,6 \\
\hline Cluster 3 & 22 & 64,7 & 12 & 35,3 & 16 & 47 & 18 & 52,9 & 21 & 61,7 & 14 & 38,2 \\
\hline
\end{tabular}

Nos três clusters, pode-se observar que a maior parte das mães não trabalha fora. Nesta amostra de crianças, a maioria frequenta a creche e, nota-se que, quanto maior a participação de outras pessoas no cuidado da criança, pior o desempenho no teste.

Os dados acima descritos sugerem, de um modo geral, que o desempenho de metade das crianças está abaixo do esperado para a idade e, durante a interação, há o predomínio do meio verbal para a comunicação. Estes achados serão minuciosamente detalhados e analisados com os efeitos da Depressão Pós-Parto nos itens a seguir.

\section{Pragmática e Depressão Pós-Parto}

Comparando-se as médias dos grupos com e sem Depressão Pós-Parto (DPP), não foram encontradas diferenças significativas dos atos comunicativos (com $\mathrm{DPP}=178$ e sem $\mathrm{DPP}=179,8)$; nem dos atos das crianças (com DPP $=88,5$ atos e sem $\mathrm{DPP}=89,6)$ e dos atos comunicativos por minuto (com DPP 5,79 e sem $\mathrm{DPP}=5,78$ ).

A maioria das crianças filhas de mães deprimidas (60\%), apresentou um desempenho abaixo do esperado para a idade, enquanto que $48 \%$ do grupo sem DPP apresentou este mesmo resultado. Registra-se então esta tendência, embora esta diferença não tenha sido significativa.

O meio comunicativo utilizado por todas as crianças (100\% das crianças de ambos os grupos), com uma média de 76,5 verbalizações no grupo com DPP e 77,7 no grupo sem DPP, seguido do gestual (93,3\% com DPP, média 13,1 e $88 \%$ sem DPP, média 8,8 ), e do vocal (93,3\% com DPP, média 4,9 e 76\% sem DPP, média 3,9). .

Quando analisadas as proporções de uso de cada meio, constatou-se que as crianças do grupo sem DPP empregaram, proporcionalmente, mais o meio verbal para se comunicar $(F=0,08 ; \mathrm{p}<0,05)$, enquanto que as crianças do grupo com DPP utilizaram, proporcionalmente, mais o meio gestual $(F=0,11$; $\mathrm{p}<0,05)$, o que sugere a influência significativa da DPP no desenvolvimento da linguagem. O meio vocal foi pouco usado por ambos os grupos.

Com relação ao contexto socioafetivo e à presença de sintomas depressivos, foi observada pequena diferença e não significativa entre as crianças dos grupos com e sem DPP. As crianças que apresentaram melhor desempenho foram aquelas cujas mães não apresentam sintomas depressivos e frequentam a creche. Apesar de não apresentarem o maior número de atos comunicativos com suas mães, estas falaram mais e utilizaram mais o meio verbal para se comunicarem. As crianças do grupo com DPP utilizaram mais gestos e vocalizações que o outro grupo.

Foi encontrada uma tendência a interação entre as variáveis DPP e trabalhar fora: as crianças, cujas mães não trabalham fora de casa e apresentavam DPP 
TABELA 3

Média e desvio padrão do desempenho das crianças no teste em relação à Depressão Pós-Parto e contexto socioafetivo

\begin{tabular}{|c|c|c|c|c|}
\hline \multirow[b]{2}{*}{ Creche } & \multicolumn{2}{|c|}{ DPP Sim } & \multicolumn{2}{|c|}{$D P P$ Não } \\
\hline & Sim & Não & Sim & Não \\
\hline $\mathrm{ACC}$ & $87,7(66,4)$ & 90,3 (17) & $92,2(31,7)$ & $82,1(25,5)$ \\
\hline $\mathrm{ACT}$ & $174(30,5)$ & $186,1(32)$ & $184,7(57,6)$ & $165,7(52,7)$ \\
\hline Vocal & $5,15(4,3)$ & $4,4(3,8)$ & $3,8(3,9)$ & $4,1(5,3)$ \\
\hline Verbal & 76,5 (29) & $76,5(20,3)$ & $79,7(29,2)$ & $72(21,6)$ \\
\hline Gestual & $11,5(8,9)$ & $16,4(11,8)$ & $9,3(9,6)$ & $7,5(8,4)$ \\
\hline Trabalha & Sim & Não & Sim & Não \\
\hline $\mathrm{ACC}$ & $82,6(22,5)$ & $92(28,6)$ & $88,2(34,5)$ & $90,7(26,9)$ \\
\hline $\mathrm{ACT}$ & $165,7(43,1)$ & $185,1(63,6)$ & $179,1(63,2)$ & $180,3(51,3)$ \\
\hline Vocal & $5,2(4,5)$ & $4,7(4)$ & $4,7(4,9)$ & $3,2(3,7)$ \\
\hline Verbal & $67,5(21)$ & $81,7(27,8)$ & 77,5 (29) & $77,8(26,5)$ \\
\hline Gestual & $18,4(12,7)$ & $10,1(6,8)$ & $7,9(7,6)$ & $9,6(10,5)$ \\
\hline Apoio & Sim & Não & Sim & Não \\
\hline $\mathrm{ACC}$ & $93,3(25,8)$ & $84,3(28,1)$ & $66,4(38)$ & $98,2(33,5)$ \\
\hline $\mathrm{ACT}$ & $192,9(55,9)$ & $165,7(58,2)$ & $178,3(54,1)$ & $197,8(65,9)$ \\
\hline Vocal & $5,3(4,4)$ & 4,4 (4) & $2,9(3,1)$ & $5,3(4,7)$ \\
\hline Verbal & $78,7(26)$ & $75,3(27,6)$ & $76,5(26,9)$ & $81,9(32,7)$ \\
\hline Gestual & $14,8(11,6)$ & $11,9(8,8)$ & $7,4(8,3)$ & $12,8(11,6)$ \\
\hline
\end{tabular}

ACC: atos comunicativos somente das crianças; ACT: atos comunicativos totais; Vocal: quantidade de vocalizações realizadas somente pelas crianças; Verbal: quantidade de verbalizações realizadas somente pelas crianças; Gestual: quantidade de gestos realizados somente pelas crianças.

obtiveram melhor desempenho, interagiram mais com suas mães e empregaram mais o meio verbal para se comunicar. Porém o grupo cujas mães trabalham fora e também apresentam sintomas depressivos, usou mais gestos e vocalizações.

\section{DISCUSSÃO}

As crianças em geral mostraram-se aquém do esperado para a idade, porém utilizaram a comunicação para transmitir uma informação, do modo que se espera das crianças de 36 meses (como, por exemplo, descrito em Zorzi e Hage, 2004, Halliday, 1975). Embora a diferença não tenha sido significativa, o grupo de crianças de mães sem sintomas depressivos obteve melhor desempenho, pois interagiu mais com sua mãe e, proporcionalmente verbalizou mais que o outro grupo, que, por sua vez, utilizou mais gestos e vocalizações.

Uma hipótese para esses resultados seria que a mãe sem indicativos de DPP estimula mais verbalmente seu bebê, através do diálogo nas brincadeiras. Para as crianças deste grupo, a creche e o apoio social recebido pela mãe parece atuar como mais estímulo de interação e desenvolvimento, pelas interações criançacriança e criança-adulto durante atividades escolares e brincadeiras. Importante salientar que a creche não parece ter o mesmo tipo de influência positiva nas crianças do grupo com DPP. Estas, por sua vez, apresentaram um melhor desempenho quando não frequentavam a creche e as mães não trabalhavam fora. Notou-se também que, utilizando gestos e vocalizações, a criança parecia chamar a atenção da mãe para si quando a mãe apresenta sintomas depressivos. Estes sintomas, de acordo com Motta et al. (2005), Silva et al. (2010) e Schmid et al. (2011), comprometem a disponibilidade cognitiva e emocional da mãe, determinando que a mãe não seja adequadamente responsiva à criança, pela falta de contingência, de engajamento e de trocas ajustadas.

Com a disponibilidade para o filho reduzida, observou-se que as crianças tentam chamar a atenção da mãe não só verbalmente, como também por outros meios (movimentos corporais, sons, etc.). Embora o desempenho tenha sido pior se comparado às crianças do grupo com DPP, estas utilizaram outras formas de se comunicar, que é explicado pelo fato de que as crianças possuem menos contato interpessoal. 
Embora os dados da presente pesquisa corroborem a equação fundamental que vem sendo proposta: DPP - prejuízo interacional mãe-criança e decorrente comprometimentos no desenvolvimento - no caso do contexto da pragmática da linguagem - também foram constatados aparentes paradoxos que esclarecem questões importantes do desenvolvimento, como a creche tender a beneficiar mais as crianças sem DPP e a mãe ficar com a criança, no caso de DPP, tender a afetar positivamente o desempenho.

A DPP não parece exercer efeito prejudicial independentemente do contexto. A mãe ter disponibilidade para a criança (não trabalhar fora, ter apoio social), proporcionou melhor desenvolvimento linguístico e, consequentemente, melhor desempenho no teste, esmo mesmo com dpp. Este resultado reitera a importância da interação mãe e filho como um processo fundamental no desenvolvimento da linguagem, como é defendido por diversos autores (Brás \& Salomão, 2002; Zorzi \& Hage, 2004; Seidl-de-Moura, 2004; Taylor, 2008; Seidl-de-Moura, 2009; Pessôa, 2009). A pragmática da linguagem envolve turnos de conversação e reciprocidade, além de representar a estrutura e funcionamento da conversação, até quando a linguagem não é utilizada. Esta interação entre crianças e adultos envolve turnos alternativos, como comportamentos de olhar, vocalizações não-verbais, expressões e gestos. Essas alternâncias de comportamentos verbais e nãoverbais evidenciam o papel do ritmo do que se chama de "conversação".

\section{CONSIDERAÇÕES FINAIS}

Pelos vários indicadores, a presente pesquisa reitera a importância da mãe no desenvolvimento da criança - sem diminuir a importância das demais pessoas do contexto familiar e nem da rede social materna e da criança. Entende-se que mães com DDP podem ser agentes do desenvolvimento, pois, mesmo com as limitações impostas pela própria DDP, se mostraram, neste estudo, provedoras de modelo linguístico para seus filhos. O apoio social para a mãe e a inserção da criança na creche, podem não ser suficientes para compensar questões ligadas à DPP. A creche, conforme os resultados desta pesquisa, deve ser relativizada quanto ser uma oportunidade de desenvolvimento equivalente para todas as crianças.

Assim, outros estudos estão em andamento dentro do Projeto Ipê, com relação ao desenvolvimento neuropsicomotor, compreensão de intencionalidade e teoria da mente, relações parentais, desenvolvimento cognitivo, disponibilidade materna, no sentido de aprofundar o conhecimento da influência da depressão pós-parto no desenvolvimento comunicativo-linguístico da criança para que propostas de intervenção sejam formuladas com objetivo de atender às necessidades específicas desta população. As limitações deste trabalho foram relacionados à problemas técnicos, como a filmagem (qualidade visual e compreensão do áudio) e dificuldade de encontrar literatura relacionada a depressão pós-parto e linguagem infantil.

\section{REFERÊNCIAS}

Andrade, C. R. F, Befi-Lopes, D. M., Fernandes, F. D. M., \& Wertner, H. F. (2000). ABFW-Teste de linguagem infantil nas áreas de fonologia, vocabulário, fluência e pragmática. Barueri: Pró-Fono.

Araújo, B. B. M. (2007). Vivenciando a internação do filho prematuro na UTIN: (re)conhecendo as perspectivas maternas diante das demandas neonatais. Dissertação de Mestrado, Universidade do Rio de Janeiro.

Arteche, A., Joormann, J., Harvey, A., Craske, M., Gotlib, I. H., Lehtonen, A., Counsell, N., \& Stein, A. (2011). The effects of postnatal maternal expression and anxiety on the processing of infant faces. Journal of affective disorders, 133, 197-203. http://dx.doi.org/10.1016/j.jad.2011.04.015

Colaço,V. F. R. (2004). Processos interacionais e a construção de conhecimento e subjetividade de crianças. Psicologia: Reflexão e Crítica, 17(3), 333-340. http://dx.doi.org/10.1590/S0102-79722004000300006

Cruz E. B. S., Simões G. L., \& Faisal-Cury, A. (2005). Rastreamento da depressão pós-parto em mulheres atendidas pelo Programa de Saúde da Família. Revista Brasileira de Ginecologia e Obstetrícia, 27, 181-188. http://dx.doi.org/10.1590/ S0100-72032005000400004

Da Silva V. A., Moraes-Santos A. R., Carvalho M. S., Martins M. L., \& Teixeira N. A. (1998). Prenatal and postnatal depression among low income Brazilian women. Brazilian Journal of Medical Biology Research, 31, 799-804. http://dx.doi.org/10.1590/ S0100-879X1998000600012

Darcy, J. M., Grzywacs, J. G., Stephens, R. L., Leng, I., Clinch, R., \& Arcury, T. A. (2011). Maternal depressive symptomatology: 16-month follow-up of infant and maternal health-related quality of life. Journal of the American Board of Family Medicine, 24(3), 249-257. http://dx.doi.org/10.3122/jabfm.2011.03.100201

Fonseca, V. R. J. R. M., Silva, G. A., \& Otta, E. (2010). Relação entre depressão pós-parto e disponibilidade emocional materna. Caderno de Saúde Pública, 26(4),738-746. http://dx.doi.org/10.1590/S0102-311X2010000400016

Halliday, M. K. (1975). Learn how to mean. Londres: Edward Arnold. 
Kersten-Alvarez, L. E., Hosman, C. M., Riksen-Walraven, J. M., Van Doesum, K. T., Smeekens, S., \& Hoefnagels, C. (2012). Early school outcomes for children of postpartum depressed mothers: comparison with a community sample. Child Psychiatry Human Development, 43, 201-218. http://dx.doi.org/10.1007/s10578-011-0257-y

Konradt C. E., Silva, R. A., Jausen, K., Vianna, D. M., Quevedo, L. A., Souza, L. D. M., Oses, J. P., \& Pinheiro, R. T. (2011). Depressão pós-parto e percepção de suporte social durante a gestação. Revista de Psiquiatria do Rio Grande do Sul, 33(2), 76-79. http://dx.doi.org/10.1590/S0101-81082011005000010

Lopes, E. R., Jansen, K., Quevedo, L. A., Vanila, R. G., Silva, R. A., \& Pinheiro, R. T. (2010). Depressão pós-parto e alterações de sono aos 12 meses em bebês nascidos na zona urbana da cidade de Pelotas/RS. Jornal brasileiro de Psiquiatria, 59(21), 88-93. http://dx.doi.org/10.1590/S0047-20852010000200002

Lucci, T., Morais, M. L. S. (2010). Relações entre depressão materna e desenvolvimento neuropsicomotor de bebês aos quatro, oito e 12 meses de vida. Resumos XII Congresso Brasileiro de Psicologia do Desenvolvimento, Brasília.

Motta, M. G., Lucion, A. B., \& Manfro, G. G. (2005). Efeitos da depressão materna no desenvolvimento neurobiológico e psicológico da criança. Revista de Psiquiatria do Rio Grande do Sul, 27(2). http://dx.doi.org/10.1590/S010181082005000200007

Murray, L., Halligan, S. L., \& Cooper, P. J. (2010). Effects of postnatal depression on mother-infant interactions and child development. Wiley: Blackwell. pp. 102-220. http://dx.doi.org/10.1002/9781444327588.ch8

Murray, L., Arteche, A., Fearon, P., Halligan, S., Croudace, T., \& Cooper, P. (2010). The effects of maternal postnatal depression and child sex on academic performance at age 16 years: a developmental approach. Journal of child Psychology and Psychiatry. 51(10), 1150-1159. http://dx.doi.org/10.1111/j.1469-7610.2010.02259.x

Nogueira, S. E. (2009). A comunicação pré-verbal. In M. L. Seidl-de-Moura, D. M. L. F. Mendes, \& L. F. Pessôa. Interação social e desenvolvimento (pp. 101-115). Rio de Janeiro: Editora CRV.

Pessôa, L.F.(2009). A fala materna e o desenvolvimento da linguagem inicial. In M. L. Seidl-de-Moura, D. M. L. F. Mendes, \& L. F. Pessôa. Interação social e desenvolvimento (pp. 117-129). Rio de Janeiro: Editora CRV.

Seidl-de-Moura, M. L. \& Ribas, A. F. P. (2004). Evidência sobre características de bebês recém-nascidos: um convite a reflexões teóricas. In M. L. Seidl-de-Moura. O bebê do século XXI e a psicologia em desenvolvimento (pp. 21-60). São Paulo: Casa do Psicólogo.

Seidl-de-Moura, M. L. (2009). Interações sociais e desenvolvimento. In M. L. Seidl-de-Moura, D. M. L. F. Mendes, \& L. F. Pessôa. Interação social e desenvolvimento (pp. 20-36). Rio de Janeiro: Editora CRV.

Schmid, B., Blomeyer, D., Buchmann, A. F., Trautmann-Villalba, P., Zimmermann, U.S., Schmidt, M. H., Esser, G., Banaschewski, T., \& Laucht, M. (2011). Quality of early mother-child interaction associated with depressive psychopathology in the offspring: A prospective study from infancy to adulthood. Journal of Psychiatric Research, 45, 1387-1394. http:// dx.doi.org/10.1016/j.jpsychires.2011.05.010

Schwengber, D. D. S. \& Piccinini, C. A. (2003). O impacto da depressão pós-parto para a interação mãe-bebê. Estudos de Psicologia, 8(3), 403-411. http://dx.doi.org/10.1590/S1413-294X2003000300007

Schwengber, D. D. S. \& Piccinini, C. A. (2005). A experiência da maternidade no contexto da depressão materna no final do primeiro ano de vida do bebê. Estudos de Psicologia, 22(2), 143-156. http://dx.doi.org/10.1590/S0103-166X2005000200004

Silva, G. A. (2008). Estudo longitudinal sobre prevalência e fatores de risco para depressão pós-parto em mães de baixa renda. Dissertação de Mestrado, Universidade de São Paulo.

Skafuzka E. (2000). Prevalência da depressão pós-parto e fatores associados em mães que deram à luz em um Hospital Universitário do Município de S. Paulo.Tese de Doutorado, Universidade de São Paulo.

Taylor, N., Donovan, W., Miles, S., \& Leavitt, L. (2009). Maternal control strategies, maternal language usage and children's language usage at two years. Journal of child language, 36(2), 381-404. http://dx.doi.org/10.1017/S0305000908008969

Thomaz, A. C. P., Lima, M. R. T., Tavares, C. H. F., \& Oliveira, C. G. (2005). Relações afetivas entre mães e recém-nascidos a termo e pré-termo: variáveis sociais e perinatais. Estudos de Psicologia, 10(1), 139-146. http://dx.doi.org/10.1590/S1413294X2005000100016

Vieira, M. L. \& Prado, A. B. (2004). Abordagem evolucionista sobre a relação entre filogênese e ontogênese no desenvolvimento infantil. In M. L. Seidl-de-Moura. O bebê do século XXI e a psicologia em desenvolvimento (pp. 155-204). São Paulo: Casa do Psicólogo.

Yamamoto, M.E.; Lopes, F.A. (2004). Dize-me o que falas e te direi o que comes: aquisição da linguagem e composição da dieta em crianças. In M. L. Seidl-de-Moura. O bebê do século XXI e a psicologia em desenvolvimento (pp. 205-228). São Paulo: Casa do Psicólogo.

Zorzi, J. L. \& Hage, S. R. (2004). PROC: Protocolo de Observação Comportamental: avaliação de linguagem e aspectos cognitivos infantis. São José dos Campos: Pulso Editorial.

\footnotetext{
Autores:

BEATRIZ SERVILHA - Doutor na Universidade de São Paulo.

VERA SILVIA RAAD BUSSAB - Doutor na Universidade de São Paulo.

Endereço para correspondência:

Beatriz Servilha

Rua Doutor Alberto Cerqueira Lima, 206 apto. 102 - Taquaral

13076-010 Campinas, SP, Brasil

E-mail: biaservilha@yahoo.com.br
}

Recebido em: 25.04 .14

Aceito em: 01.09.14

Psico, Porto Alegre, PUCRS, v. 46, n. 1, pp. 103-111, jan.-mar. 2015 